\title{
MICROSTRUCTURAL MODELLING OF FATIGUE INITIATION IN ALUMINIUM-BEARING ALLOYS
}

\author{
S. SYNGELLAKIS ${ }^{1}$, M.S. ALI ${ }^{2} \&$ P.A.S. REED ${ }^{3}$ \\ ${ }^{1}$ Wessex Institute of Technology, UK. \\ ${ }^{2}$ TWI Ltd, UK. \\ ${ }^{3}$ University of Southampton, UK.
}

\begin{abstract}
Microstructural fatigue initiation in $\mathrm{Al}-\mathrm{Sn}$-Si-Cu-Ni bearing lining alloys is reported and investigated. The secondary phases of such alloys comprise fine and relatively few $S n$ and $S i$ particles as well as a large number of hard $\mathrm{AlNi}_{3}$-type intermetallics, frequently encapsulated within soft $\mathrm{Sn}$ layers. During fatigue tests, these particles were observed to initiate short fatigue cracks. Through elasto-plastic finite element analysis of ideal microstructures but with realistic geometric and mechanical property data, critical values of key stress and strain components within the matrix, the $S n$ layers, and the particles were predicted and linked to microstructural features associated with observed fatigue initiation. These modelling results indicate the extent to which either the hydrostatic stresses or plastic shear strains may be responsible for fatigue crack initiation in the $S n$ layers, as well as the optimum microstructural characteristics that would minimise tensile stresses, which are responsible for brittle particle fracture. Keywords: Aluminium alloys, bearing linings, fatigue initiation, micromechanics, plasticity, secondary phases.
\end{abstract}

\section{INTRODUCTION}

The lining surface of journal bearings may suffer fatigue damage under elasto-hydrodynamic lubrication conditions arising during engine operation. In practice, fatigue resistance of various plain bearing designs is measured as lifetimes represented by the number of runs to failure on an accelerated test rig under well controlled loading conditions associated with rather complex states of stress and strain [1]. A more well-defined fatigue evaluation approach was adopted in more recent works [2,3] according to which fatigue life is assessed using a deflection-based failure criterion linked to three-point bend loading. The overall fatigue life of such a multilayered material system comprises the loading cycles to small crack initiation and those responsible for subsequent surface as well as subsurface crack growth until failure. Cracks, once initiated, grow on the lining surface during fatigue life until their subsurface growth is retarded due to the presence of a harder backing steel layer. Unless cracks coalesce, their surface growth appears to cease until the specimen deflection reaches the value set as the failure criterion. Earlier work has shown that fatigue initiation is linked to microstructural heterogeneities such as grain boundaries [4], inclusions [5], defects [6] and secondary phase particles [7,8]. The bearing alloys investigated during previous [2] and current work are complex multiphase $\mathrm{Al}$ alloys in which secondary-phase particles are distinguishable at microscale level and scattered throughout the matrix.

Microstructural fatigue initiation studies carried out on two lining alloys, $\mathrm{Al}-12 \mathrm{Sn}-4 \mathrm{Si}-1 \mathrm{Cu}$ and $\mathrm{Al}-20 \mathrm{Sn}-1 \mathrm{Cu}-0.25 \mathrm{Mn}$, designated AS1241 [9] and AS16 [2], respectively, showed that coarser $\mathrm{Sn}$ and $\mathrm{Si}$ particles were responsible for early initiation of microstructurally short fatigue cracks. It was also shown that the actual initiation behaviour was associated with a complex combination of particle distribution and geometries [9]. Previous work on Al-6.5Sn-2.5 $\mathrm{Si}-1 \mathrm{Cu}-1 \mathrm{Ni}-0.25 \mathrm{Mn}$, designated AS20S, showed improved overall fatigue life $[3,10]$ compared to that of the AS16 and AS1241 systems. This is a more recently employed lining alloy with 
finer and fewer $S n$ particles as well as occasional $S i$ particles. The loading cycles to small crack initiation accounted for 15-20\% of its overall fatigue life. A detailed study of microstructural fatigue initiation carried out on AS2OS showed that $\mathrm{AlNi}_{3}$ type intermetallic compounds, some of which are encapsulated within soft $S n$ films, were responsible for early short-crack initiation. Using Eshelby's analytical approach [11] and assuming intermetallics to be ellipsoidal elastic particles embedded in an $A l$ matrix, stress calculations showed that stiffer, more brittle intermetallic particles carried higher tensile stresses, and hence, are more prone to fracture as compared to the softer surrounding matrix [10].

In this paper, a detailed interpretation of fatigue initiation in the AS20S lining is attempted with the help of micromechanical elasto-plastic finite element (FE) modelling through which a more complex pattern of interacting $S n$ and intermetallic phases is analysed. The FE analysis will benefit from comparisons with relevant experimental data, thus leading to a greater understanding of the influence of various geometrical factors responsible for early fatigue initiation in such alloys.

\section{EXPERIMENTAL WORK}

Specimens of the AS20S material system in the form of tri-layer flat bars were fatigue tested under the three-point bend configuration shown in Fig. 1. A validated elasto-plastic FE simulation of the test provided reliable estimates of the maximum tensile stress and plastic strain on the lining surface at the middle of the bar, directly opposite to the applied load. Both uninterrupted and interrupted tests were conducted in order to compare total lifetimes with the load cycles corresponding to short-crack initiation and growth regimes. The interrupted tests used frequent acetate replications to monitor crack initiation and growth behaviour at a load corresponding to a maximum lining plastic strain level of 0.0064 . For one particular specimen, subjected to the same maximum plastic strain, the test was stopped as soon as microstructurally short fatigue cracks appeared on the lining surface of the specimen, observed under optical microscope. A large number of secondary electron images (SEI) were obtained from the cracked surface to examine qualitatively the type of particles participating in early fatigue initiation. The finite body tessellation technique (FBT) [12] was used to investigate quantitatively the geometrical features of short-crack initiating particles.

Figure 2 shows scanning electron microscope (SEM) images of lining surfaces of fatigued flat bar specimens. Comparison of AS20S with AS1241 and AS16 images [2] confirms that the microstructure of the AS20S lining is more complex in terms of particle shapes and

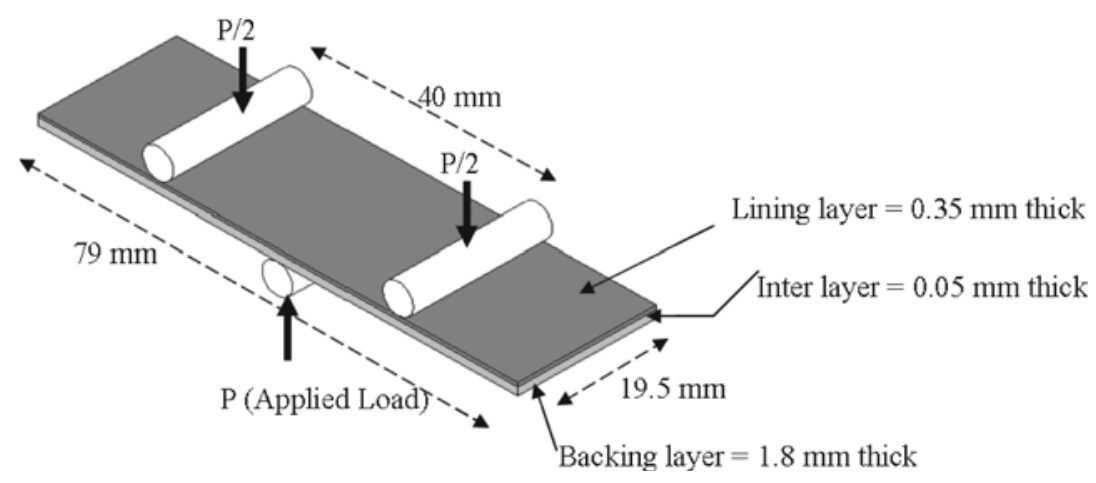

Figure 1: Three-point bend test configuration for the AS20S flat bar specimen. 


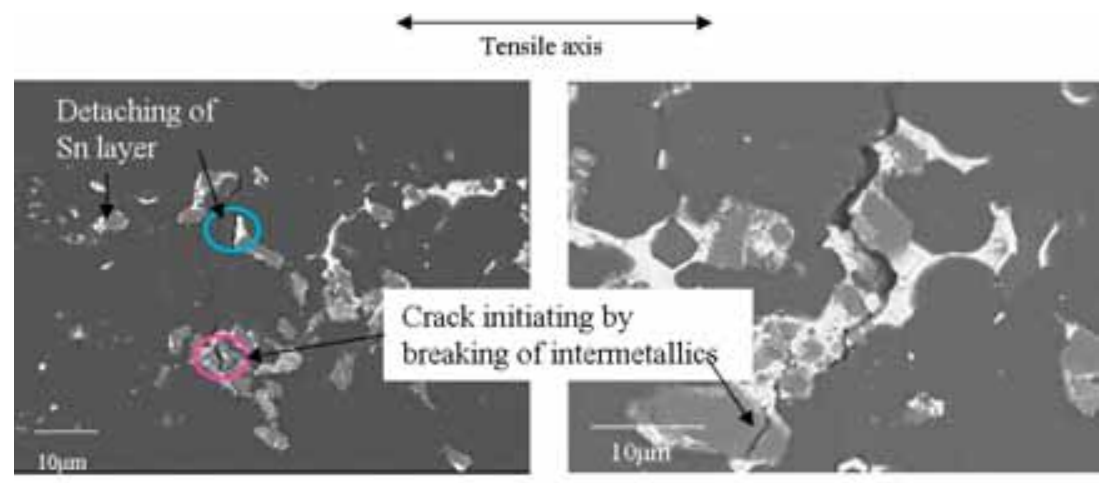

(a)

(b)

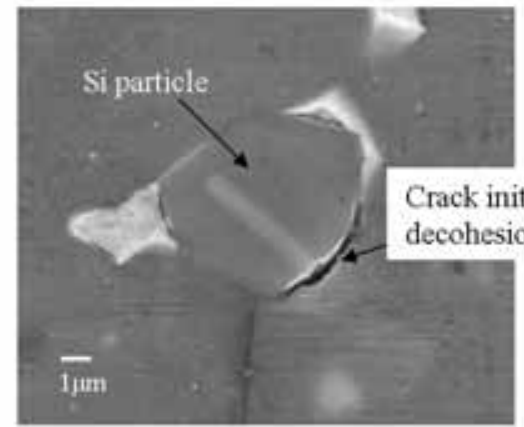

(c)

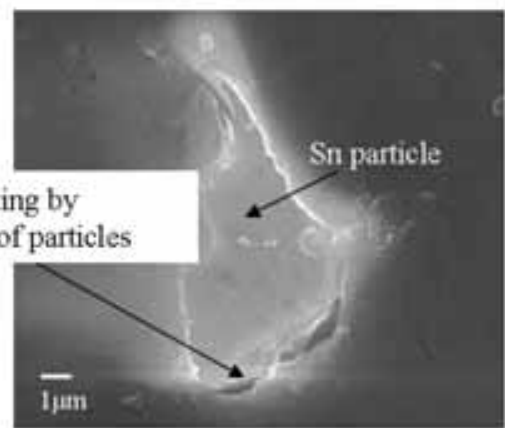

(d)

Figure 2: SEM images of fatigued lining surfaces showing various particles initiating short cracks: (a) and (b) AS20S; (c) AS1241; (d) AS16.

morphology. It is noted that the short fatigue crack initiation process depends on different properties of the individual particles; moreover, the particle perimeters are not well defined, making identification of such a process difficult. Secondary-phase particles exist as aggregates of $\mathrm{Sn}$ and $\mathrm{AlNi}_{3}$ type intermetallics rather than individual, discrete particles.

The majority of crack initiation events was due to fracturing of intermetallics, although detachment of $S n$ layers from intermetallics and decohesion of $S n$ particles from the $A l$ matrix was also quite evident. Geometrical features of crack initiating and non-initiating particles measured using the FBT method are given in Table 1. The FBT parameters listed in that table are the area of individual sectioned particles (objects) $A_{o}$, the aspect ratio of that area $r_{o}$, the angle between the area's longest chord and the loading axis $\beta_{o}$, and the mean interfacial distance between a particle and all its neighbours $d_{m}$. It is evident from that table that crack initiation in both intermetallic and $S n$ particles can be linked to larger size and wider scatter of such particles since the values of $A_{o}$ and $d_{m}$ for the initiating particles are greater than those for the non-initiating ones. With regard to $S n$ layers, their detachment appears to be linked to greater alignment with the loading axis and, rather weakly, to the thinness of as well as the proximity between layers.

The dominant crack initiation mechanisms in the AS20S lining shown in Fig. 2 can be explained in terms of the compliance mismatch between the $A l$ matrix, the $S n$ layers, and the intermetallics, leading to significant load transfer to the intermetallics. This was confirmed by 
Table 1: FBT-measured features for various short-crack initiating particles in the AS20S lining (number of particles in brackets).

\begin{tabular}{lcccccc}
\hline & \multicolumn{2}{c}{ Intermetallics } & \multicolumn{2}{c}{$S n$ particles } & \multicolumn{2}{c}{$S n$ layers } \\
\cline { 2 - 7 } Features & $\begin{array}{c}\text { Initiating } \\
(293)\end{array}$ & $\begin{array}{c}\text { Non-initiating } \\
(333)\end{array}$ & $\begin{array}{c}\text { Initiating } \\
(98)\end{array}$ & $\begin{array}{c}\text { Non-initiating } \\
(345)\end{array}$ & $\begin{array}{c}\text { Initiating } \\
(167)\end{array}$ & $\begin{array}{c}\text { Non-initiating } \\
(334)\end{array}$ \\
\hline$A_{o}(\mu \mathrm{m} 2)$ & $45 \pm 29$ & $24 \pm 15$ & $37 \pm 12$ & $17 \pm 15$ & $17 \pm 10$ & $21 \pm 11$ \\
$r_{o}$ & $1.5 \pm 0.41$ & $1.5 \pm 0.39$ & $1.62 \pm 0.37$ & $1.6 \pm 0.47$ & $1.97 \pm 0.59$ & $1.93 \pm 0.66$ \\
$\beta_{o}(\mathrm{rad})$ & $0.8 \pm 0.46$ & $0.86 \pm 0.41$ & $0.93 \pm 0.50$ & $0.95 \pm 0.38$ & $0.50 \pm 0.39$ & $0.93 \pm 0.39$ \\
$d_{m}(\mu \mathrm{m})$ & $66 \pm 25$ & $43 \pm 23$ & $130 \pm 64$ & $56 \pm 32$ & $62 \pm 29$ & $82 \pm 43$ \\
Total area & 0.008 & 0.0096 & 0.0023 & 0.0041 & 0.0032 & 0.0018 \\
fraction & & & & & & \\
\hline
\end{tabular}

a two-dimensional version of the Eshelby solution for an elastic ellipsoidal particle embedded in an elastic continuum [11], which was applied to an elliptical inclusion, representing an $S n$ or intermetallic particle, in an $\mathrm{Al}$ matrix [10].

\section{MICROMECHANICAL ELASTIC ANALYSES}

Fatigue initiation at the microscale level can be attributed to high stresses and strains developing at various critical locations under the applied load. Fracture and fatigue in metal matrix composites are traditionally associated with high levels of tensile stress and plastic strain [5,13], respectively. It was also hypothesised that the normal component of the interfacial traction $p_{n}$ may be responsible for void nucleation in short-fibre-reinforced aluminium alloys [14]. It was subsequently suggested [15] and experimentally confirmed [16] that a crack is likely to initiate due to interfacial decohesion of the secondary particles when the maximum hydrostatic component $\sigma_{h}$ of the matrix stress state reaches a critical value. Micromechanical analyses were thus performed in order to assess the maximum values and respective locations of these critical parameters for various microstructural patterns and loading orientations.

A bi-material model was initially analysed using the exact elastic solution of the elliptical inclusion problem [17], which is the two-dimensional equivalent to the Eshelby problem [11]. As mentioned above, the purpose of this analysis was to not only provide some qualitative information on the magnitude and location of critical stresses under elastic conditions but also to serve as a means of validation of the subsequently built FE model. The elliptic inclusion was meant to represent either a hard intermetallic or a soft $S n$ particle embedded in an infinite, mechanically homogeneous and isotropic AS20S matrix. The analysed geometry is a special case of the more general one shown in Fig. 3, which includes a layer of uniform thickness $t$ around the particle as well as a counter-clockwise rotation $\alpha$ of the particle $x^{\prime}-y^{\prime}$ axes relative to the loading $x-y$ axes. This more complex geometry was subsequently analysed by FE assuming elasto-plastic properties for the layer and the matrix. The elastic analysis was performed with $t=\alpha=0$, that is, without an encapsulating layer and the particle $y^{\prime}$ axis aligned with the loading $y$ axis. The Cartesian coordinates of the elliptical interface are related to the elliptical coordinate $\theta$ by

$$
x^{\prime}=a \cos \theta, y^{\prime}=b \sin \theta
$$




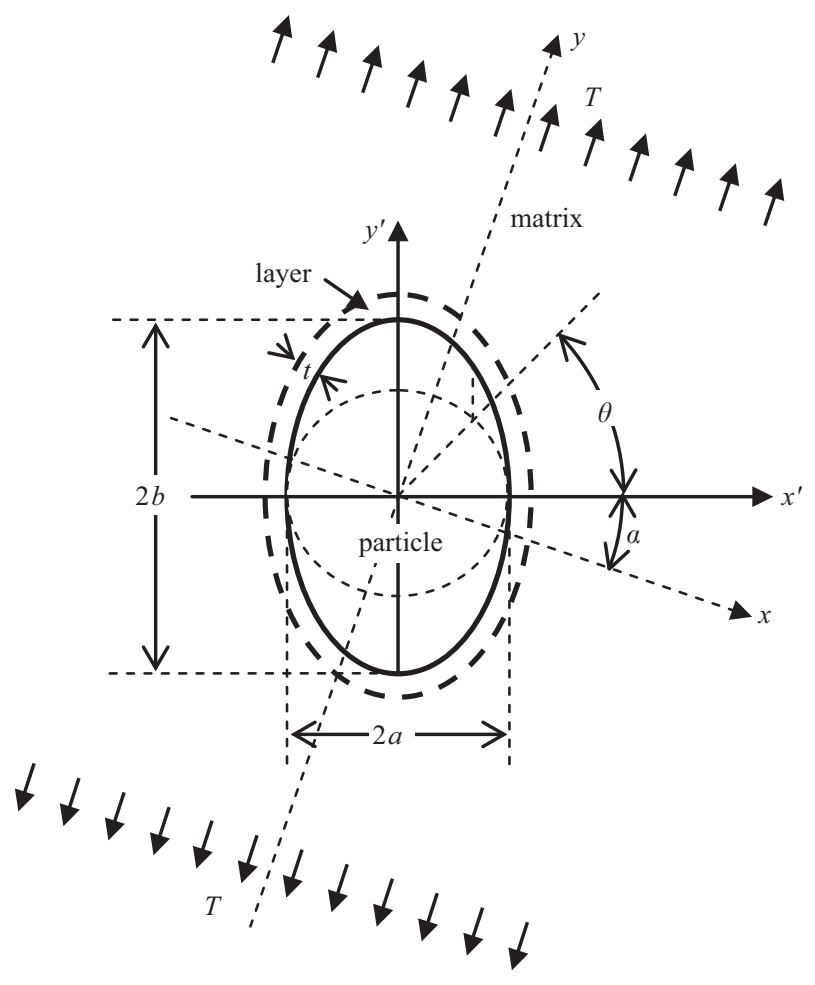

Figure 3: Simplified two-dimensional micromechanical model.

where $a$ and $b$ are the ellipse semi-axes along the $x^{\prime}$ and $y^{\prime}$ directions, respectively.

The predicted state of stress in the particle is uniform and the maximum tension $\sigma_{p}$ is aligned with the loading ( $y$ ) axis; $\sigma_{p}$ is also equal to the maximum normal interface traction $p_{n}$. The state of stress in the matrix is non-uniform, but all critical values are reached at the particle-matrix interface. In the case of hard inclusions, the maximum tensile stress $\sigma_{m}$ and shear stress $\tau_{m}$ develop at a certain angular coordinate, represented by $\theta_{\sigma m}$ and $\theta_{\tau m}$, respectively. The stress in the loading direction, $\sigma_{y}$, indicates the degree of load transfer from the matrix to the particle and its minimum value develops at $\theta=0^{\circ}$; the maximum hydrostatic stress $\sigma_{h}$ develops at $\theta=90^{\circ}$. In the case of soft inclusions, $\sigma_{m}$ is equal to maximum $\sigma_{y}$, while both $\tau_{m}$ and $\sigma_{h}$ develop at $\theta=0^{\circ}$.

The variation in these stresses with the particle aspect ratio $r=b / a$ was obtained using the values of elastic material properties listed in Table 2, which were retrieved from various literature sources $[18,19]$. Consistent values for the Young's moduli of the secondary phases were obtained by a characterisation method based on nano-indentation test data [20]. A remote tension $T=100 \mathrm{MPa}$, aligned with the $y$-axis, was applied. The results are presented in Figs 4 and 5 for the hard and soft inclusion, respectively. The load transfer from the softer to the harder phase is evident in these graphs for both cases. This transfer appears to increase with particle aspect ratio in the case of hard inclusion, while it decreases in the case of the soft one. It is worth noting the very high tension generated locally in the matrix by the hard inclusion. Both particle fracture or interface void nucleation are, therefore, possible modes of failure in this case. 
Table 2: Elasto-plastic properties used in finite element modelling.

\begin{tabular}{lccccc}
\hline Phase & $\begin{array}{c}\text { Young's } \\
\text { modulus } E(\mathrm{GPa})\end{array}$ & $\begin{array}{c}\text { Poisson's } \\
\text { ratio } v\end{array}$ & $\begin{array}{c}\text { Yield stress } \\
\sigma_{Y}(\mathrm{MPa})\end{array}$ & $\begin{array}{c}\text { Ultimate stress } \\
\sigma_{u}(\mathrm{MPa})\end{array}$ & $\begin{array}{c}\text { Elongation } \\
(\%)\end{array}$ \\
\hline Al lining & $70^{\mathrm{c}}$ & $0.33^{\mathrm{c}}$ & $66^{\mathrm{a}}$ & $166^{\mathrm{a}}$ & $28^{\mathrm{a}}$ \\
$S n$ & $45^{\mathrm{c}}$ & $0.33^{\mathrm{c}}$ & $43^{\mathrm{b}}$ & $60^{\mathrm{c}}$ & $40^{\mathrm{c}}$ \\
Intermetallic & $197^{\mathrm{c}}$ & $0.32^{\mathrm{c}}$ & N/A & N/A & N/A \\
\hline
\end{tabular}

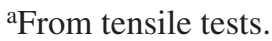

${ }^{b}$ From nano-hardness tests.

${ }^{\mathrm{c}}$ From reference literature $[18,19]$.

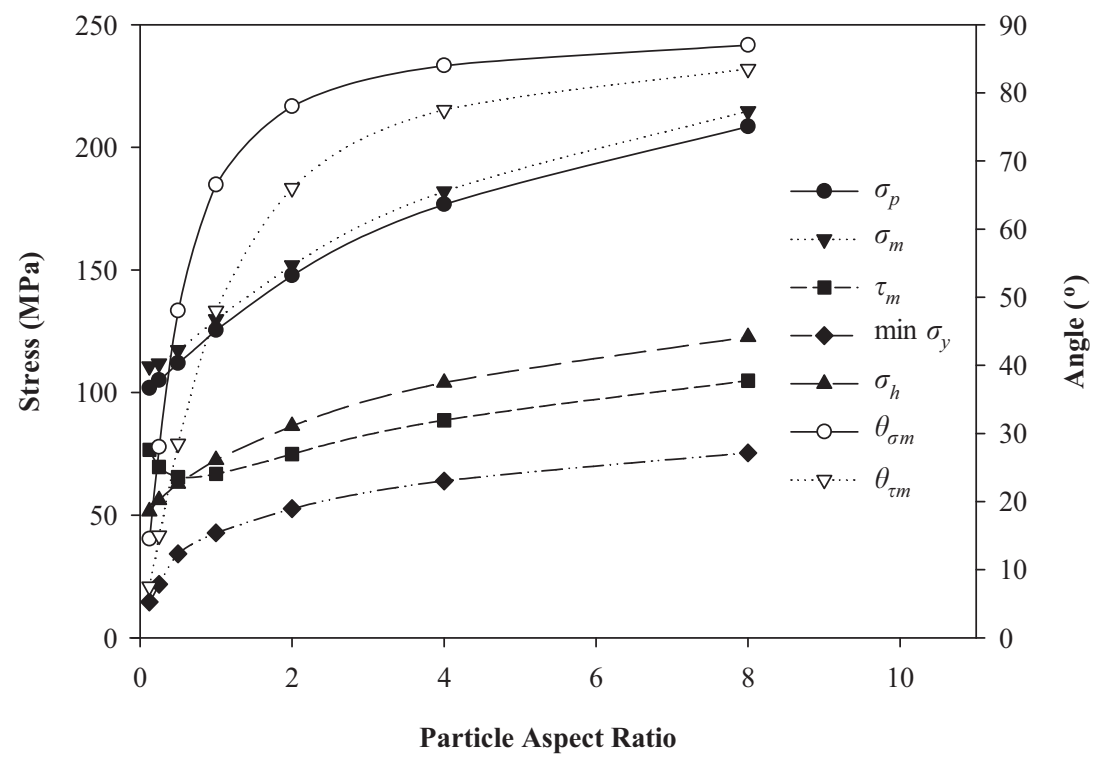

Figure 4: Exact elastic analysis predictions of critical stresses in matrix and embedded hard particle $\left(T=100 \mathrm{MPa}, \alpha=0^{\circ}\right)$.

FE modelling of the same problem was undertaken using the general-purpose code ANSYS [21]. As already pointed out, the main purpose of the elastic FE analysis was to establish its reliability in predicting the extreme stress values in the particle and the matrix. The model comprised a single elliptical particle at the centre of a square-shaped matrix domain, and it was meshed using eight-node quadrilateral elements. The material properties of Table 2 were again used and tension $T=100 \mathrm{MPa}$ applied in the $y$ direction. FE results were obtained for various meshes, matrix sizes and boundary conditions. The requirement on these results to be in good agreement with the exact analytical ones presented in Figs 4 and 5 led to a model with 144 elements along the particle-matrix interface, a particle to matrix area ratio of approximately $0.6 \times 10^{-4}$ and free of traction along the sides of the square matrix domain parallel to the loading direction. For values of the aspect ratio $b / a$ close to 1 , this model provided results with less than $1 \%$ difference from those of the exact analytical solution. This error rose to $3-4 \%$ for $r=0.125$, which was the lowest aspect ratio adopted. This level of 


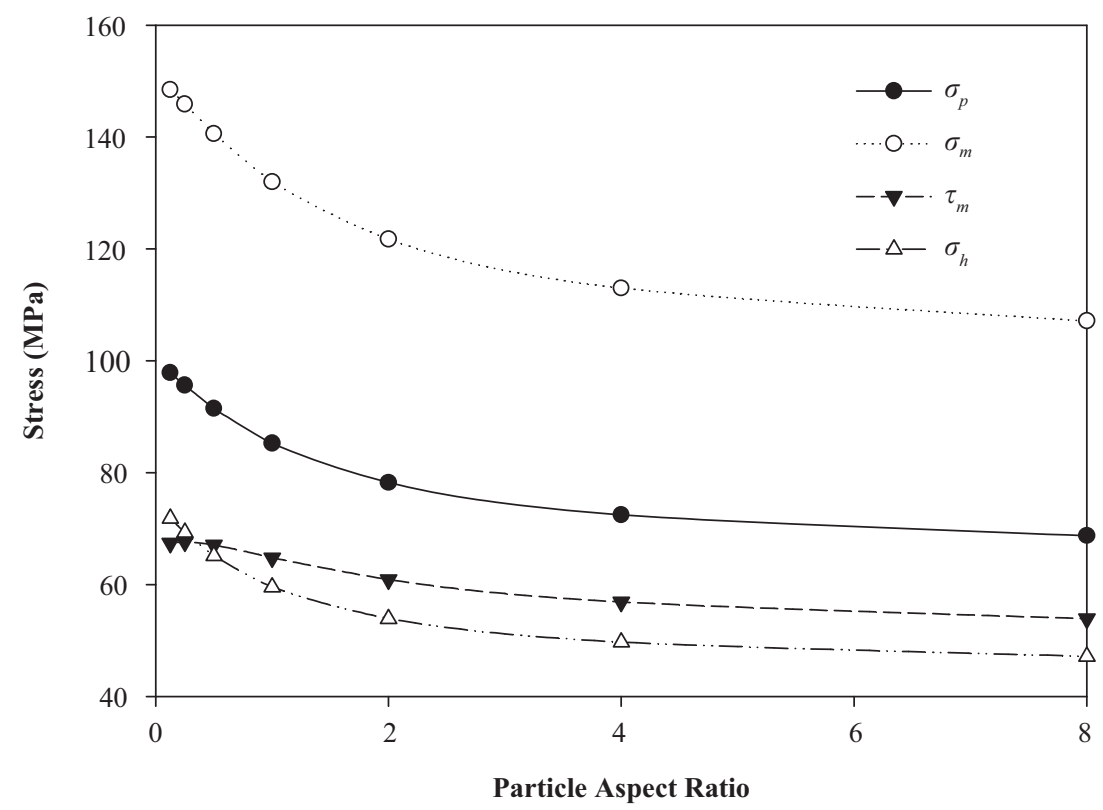

Figure 5: Exact elastic analysis predictions of critical stresses in matrix and embedded soft particle $\left(T=100 \mathrm{MPa}, a=0^{\circ}\right)$.

accuracy was considered acceptable for the purposes of the subsequent parametric studies. It was assumed, in other words, that the same accuracy would be achieved by elasto-plastic multi-domain models with similar features to those adopted in the elastic FE analysis.

\section{ELASTO-PLASTIC FE ANALYSIS}

In the AS20S lining, apart from microscale fracture of intermetallics, the detachment of soft $\mathrm{Sn}$ layers from hard intermetallics (or Si particles occasionally) was quite evident. $S n$ is also softer than the $\mathrm{Al}$ matrix. This is a complex assembly of three materials, whose response to applied load has to be systematically assessed before attempting to link it with an observed fatigue initiation process. Thus, parametric investigations were carried out to study the sensitivity of critical stresses and strains to the thickness $t$ of surrounding $S n$ layer, the orientation $\alpha$ of the encapsulated particle relative to the loading direction, and its aspect ratio $r=b / a$. The variation in these parameters was consistent with the ranges obtained from the FBT measurements given in Table 1. It should be noted that $\alpha$ is the idealised equivalent to $\beta_{o}$ for aspect ratios greater than 1 .

The adopted generic FE model was essentially the same as the one built for the elastic analysis, with the addition of a thin strip of uniform thickness $t$, representing the $S n$ layer, around the hard intermetallic particle, as shown in Fig. 3. Particular attention was given to the meshing of the $S n$ layer. Initially applied, automatic, so-called 'free' meshing produced patterns of irregularly shaped elements; such patterns were changing with increasing layer thickness. As a result, the initial output did not appear to converge to stable values with increased mesh refinement. Thus, a regular, so-called 'mapped' mesh was built comprising quadrilaterals with aspect ratio close to 1 and a constant number of elements though the layer thickness. Samples of the final layer meshes that provided convergent and smoothly varying output are shown in Fig. 6a-c for three values of $t /(2 b)$. 


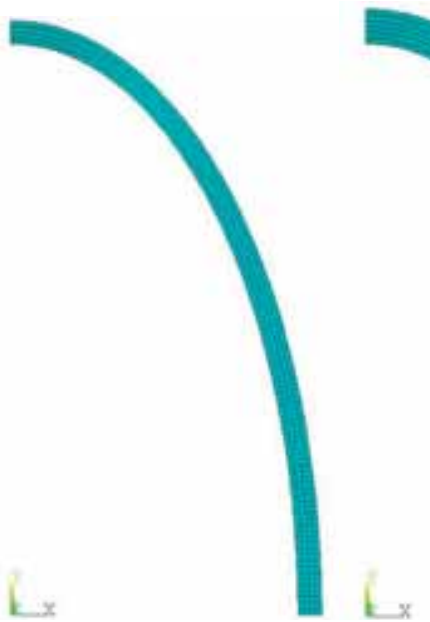

(a)

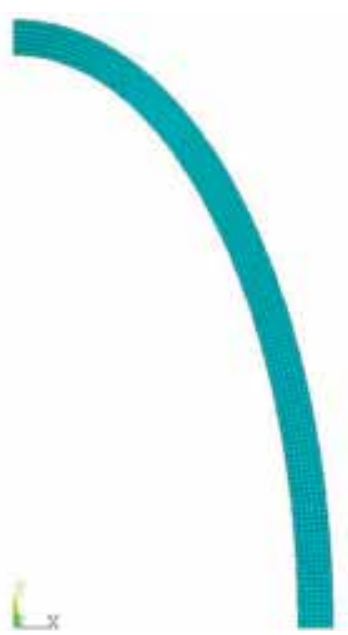

(b)

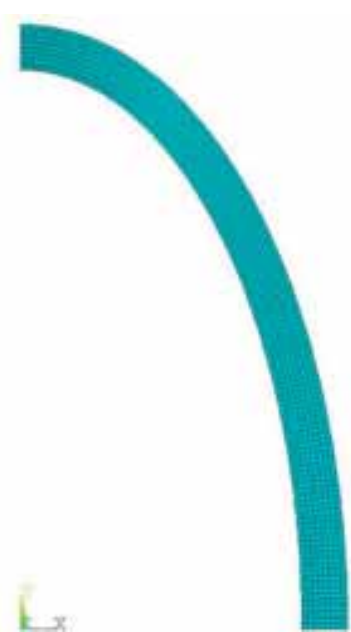

(c)

Figure 6: Meshed Sn layers for (a) $t /(2 b)=0.02$, (b) $t /(2 b)=0.03$, (c) $t /(2 b)=0.04$.

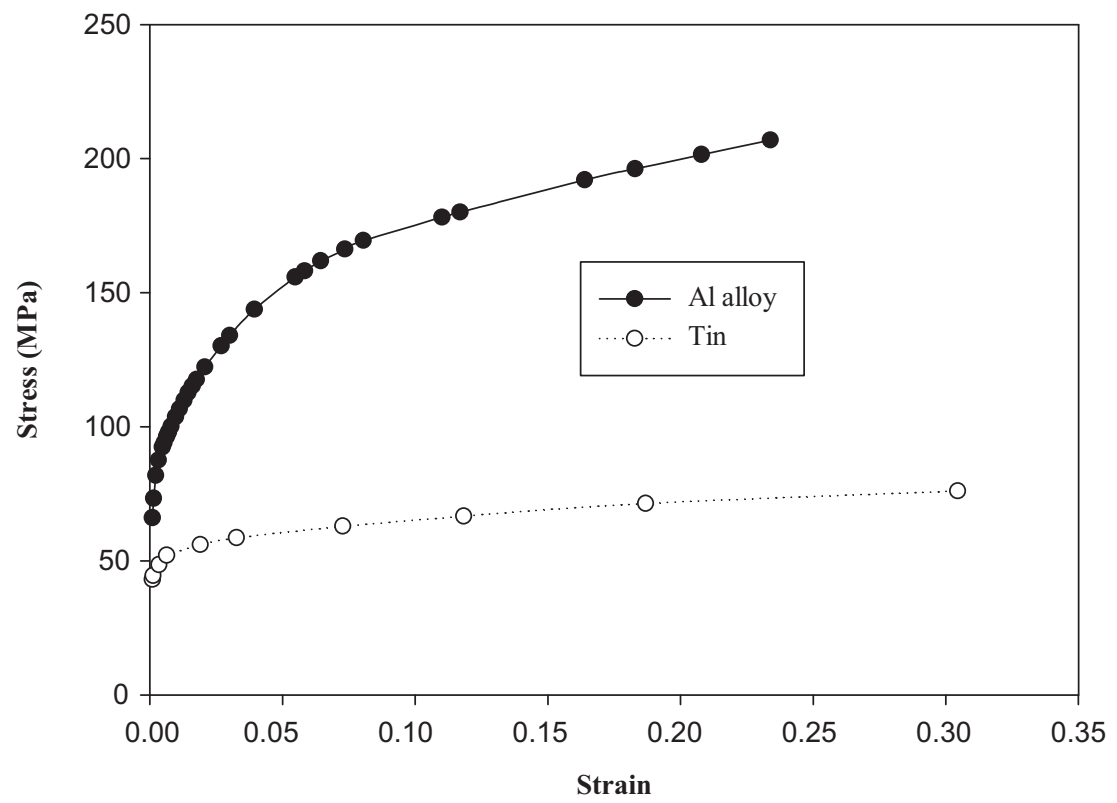

Figure 7: Stress-strain data used in elasto-plastic FE modelling.

Due to the small volume fraction $V_{f}$ of hard particles (measured $V_{f} \cong 2 \%$ according to the FBT) and the presence of counteracting soft $S n$ phase, the elasto-plastic properties of the $A l$ matrix were not expected to differ significantly from those of the three-phase composite. This was confirmed by aggregate property estimates based on a simple micromechanical model [22]. For this reason, the model did not include a pure $A l$ core, embedded in the matrix and surrounding the $S n$ layer. The intermetallic particle was assumed to remain elastic while the adopted $\sigma-\varepsilon$ flow curves for $A l$ alloy and $S n$ are shown in Fig. 7. These curves are consistent with the elasto-plastic properties of 
these two materials listed in Table 2. The $A l$ data are based on output from tensile tests on monolithic AS20S lining specimens; those for $S n$ reflect the behaviour of a coating around the hard particles in the AS20S lining whose form has emerged as a result of rolling and heat treatment operations during bearing manufacture. The applied remote tension $T=100 \mathrm{MPa}$ produced plastic strains in the $\mathrm{Al}$ matrix comparable to those predicted in the bearing lining by the FE simulation of the three-point bend test shown in Fig. 1.

It is recognised that the tri-material FE model, based on the material data listed in Table 2 and shown in Fig. 7, may not perfectly reflect the true mechanical behaviour of the constituents of the AS20S lining alloy. However, the purpose of this model was to understand the distribution of microfields of stresses and strains at various locations of a system comprising three materials of widely varying mechanical properties that may explain the possible causes of fatigue initiation in the AS20S lining. Since the stress state in the middle of the multilayered flat bar is essentially tri-axial, plane strain conditions were adopted in the FE models. As a consequence, the hydrostatic pressure would be numerically overestimated and the plastic strain underestimated on the surface of the specimens where plane stress conditions apply.

\section{RESULTS AND DISCUSSION}

\subsection{Effect of $S n$ layer thickness}

The thickness of the encapsulating $S n$ layer was varied from 0 to $10 \%$ of the particle major axis, since within the microstructure of the actual sample, the thickness of the encapsulating $S n$ layers was observed to be falling within that range. Typical contour plots of the most important output variables are presented in Fig. 8. These results were obtained for $r=2$ (hence, $2 b$ is the major axis of the ellipse) and $a=0$ (hence, the particle major axis is aligned with the tensile axis). Similar stress and strain patterns were obtained from all analyses with various $S n$ layer thicknesses. Figure 8(a) confirms the nonuniformity of the tensile stress

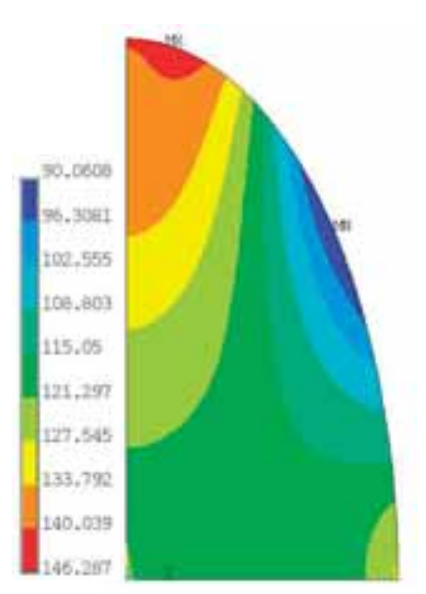

(a)

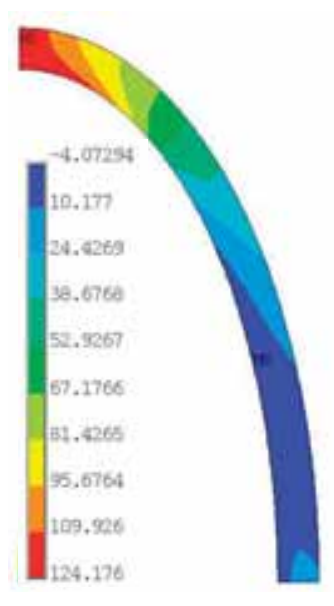

(b)

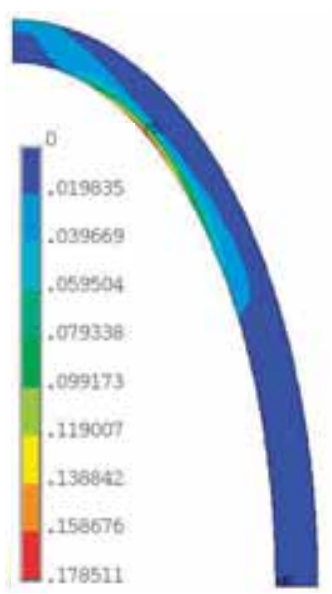

(c)

Figure 8: (a) Maximum tensile stress in particle, (b) hydrostatic stress in $S n$ layer, and (c) maximum plastic shear strain in the $S n$ layer $[t /(2 b)=0.04, T=100 \mathrm{MPa}, r=2$, $a=0^{\circ}$. 


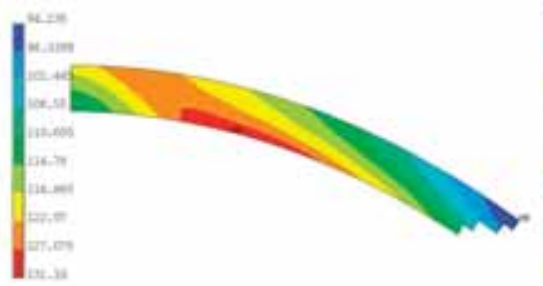

(a)

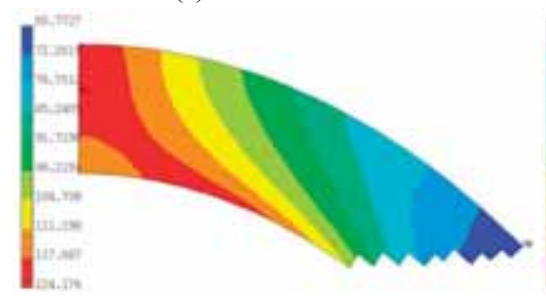

(c)

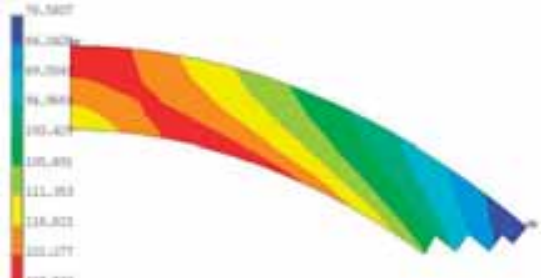

(b)

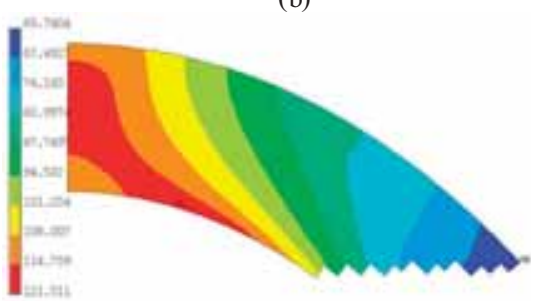

(d)

Figure 9: Location of maximum hydrostatic stress in the $S n$ layer: (a) $t /(2 b)=0.01$, (b) $t /(2 b)=0.02$, (c) $t /(2 b)=0.04$, (d) $t /(2 b)=0.05\left(T=100 \mathrm{MPa}, r=2, a=0^{\circ}\right)$.

within the particle when the surrounding material deforms elasto-plastically; its maximum value lies at the particle-layer interface, close to $\theta=90^{\circ}$. Brittle fracture is therefore likely to originate from that location for this particular particle aspect ratio.

The FE analyses predict that the location of the maximum hydrostatic stress changes with varying layer thickness; this is more clearly illustrated in Fig. 9. It is noted there that $\sigma_{h}$ moves from the particle-layer interface and $\theta<90^{\circ}$ for $t /(2 b)=0.01$ [Fig. 9(a)] to layer-matrix interface and $\theta=90^{\circ}$ for $t /(2 b)=0.02-0.03$ [Fig. 9(b)], then within the layer for $t /(2 b)=0.04$ [Fig. 9(c)] and eventually back to the particle-layer interface and $\theta<90^{\circ}$ for $t /(2 b) \geq 0.05$ [Fig. 9(d)]. In contrast, the maximum plastic shear strain lies always at the particle-layer interface, as shown in Fig. 8(c). Combining these predictions with a careful monitoring of crack initiation sites may help identify the more relevant indicator of particle-matrix decohesion in such a tri-material system. Figure 2(a) and (b) indicate that there is no a clear pattern of crack initiation in the $S n$ layers; this is consistent with the FE predictions for the maximum hydrostatic stress, thus pointing to the latter as the more likely failure parameter.

Figure 10 shows how variation in the thickness of $S n$ layer affects the predicted maximum tension $\sigma_{p}$ in the particle, as well as the maximum hydrostatic stress $\sigma_{h}$ and the maximum plastic shear strain $\gamma_{p l}$ in both $S n$ layer and $A l$ lining of the tri-material system. It is evident from Fig. 10 that the amplification of $\sigma_{\mathrm{p}}$ relative to the applied tension is significant over the whole range of layer thickness reaching a maximum of around $50 \%$ at low layer thicknesses. This is slightly higher than the $47.7 \%$ predicted by the purely elastic solution for the same particle aspect ratio $(r=2)$ and can be attributed to plasticity, which weakens the surrounding layer and matrix, and thus enhances the load transfer to the particle. The likelihood of particle brittle fracture is therefore enhanced for layer thicknesses of less than $2 \%$ of the major axis, since $\sigma_{p}$ amplification appears to peak within that range. Further increase in the encapsulating $S n$ layer thickness causes the value of $\sigma_{p}$ to drop gradually to a safer level. Therefore, a thick $S n$ layer has a protective role against particle failure. In contrast, maximum load transfer to the particle is associated with a very thin $S n$ layer, and this is consistent with experimental observations of individual intermetallic particle fracture. 


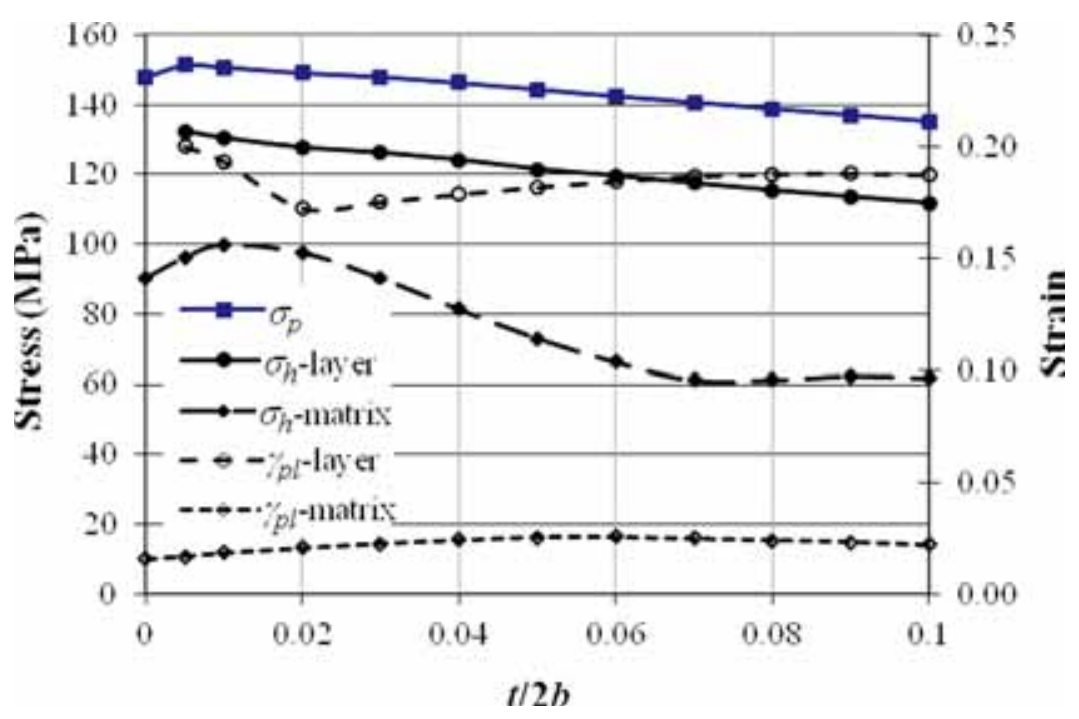

Figure 10: Critical stresses and strains versus $\operatorname{Sn}$ layer thickness $\left(T=100 \mathrm{MPa}, r=2, \alpha=0^{\circ}\right)$.

As already mentioned, the high hydrostatic stresses and localised plastic strains are considered possible causes of interface void nucleation, from which fatigue cracks may initiate. It is evident from Fig. 10 that these components are significant and predominant in the $S n$ layer; therefore, such a failure is predicted to originate from there, and it is more likely to occur for very small $S n$ layer thicknesses. This is consistent with the observed detachment of very thin $S n$ layers during the investigation of fatigue initiation in the $A l$ lining. It is also interesting to consider the ratio of the average FBT-measured area of $S n$ layers to that of the respective encapsulated intermetallics by referring to Table 1. It is noted that this ratio is considerably higher for non-initiating than initiating particle-layer combinations.

\subsection{Effect of particle orientation}

Figure 11 shows the effect of varying the particle orientation relative to the direction of the applied tension. The angle $a$ between the particle $y^{\prime}$ axis and the loading direction, as defined in Fig. 3, is the ideal equivalent of the particle object angle $\beta$, whose average is $49.3^{\circ}$ with a standard deviation of $23.5^{\circ}$, according to Table 1 . Thus, $\alpha$ was varied between $0^{\circ}$ and $90^{\circ}$ while keeping $r$ and layer thickness constant at 2 and $0.04 b$, respectively (thus, $2 b$ remained the particle major axis). Again, it is clear that fatigue failure is unlikely to originate from the matrix whatever the particle orientation. According to the stress predictions of the present elasto-plastic analysis, particle fracture and void nucleation in the layer are more likely when the major particle axis is aligned with the loading axis $(\alpha=0)$. The FBT measurements of the intermetallic object angles $\beta_{o}$ given in Table 1 do indeed show a small drop in this angle from $0.86 \mathrm{rad}\left(49.3^{\circ}\right)$ for non-initiating particles to $0.8 \mathrm{rad}\left(45.8^{\circ}\right)$ for the initiating ones.

In contrast to the maximum hydrostatic stress, which appears to drop monotonically as $\alpha$ changes from $0^{\circ}$ to $90^{\circ}$, the maximum plastic shear strain in the layer peaks twice, at $\alpha \cong 12^{\circ}$ and $a \cong 78^{\circ}$. There are no FBT results linking hard particle orientation with void nucleation in the soft layer; hence, it is not possible to suggest that either of the two parameters might be 


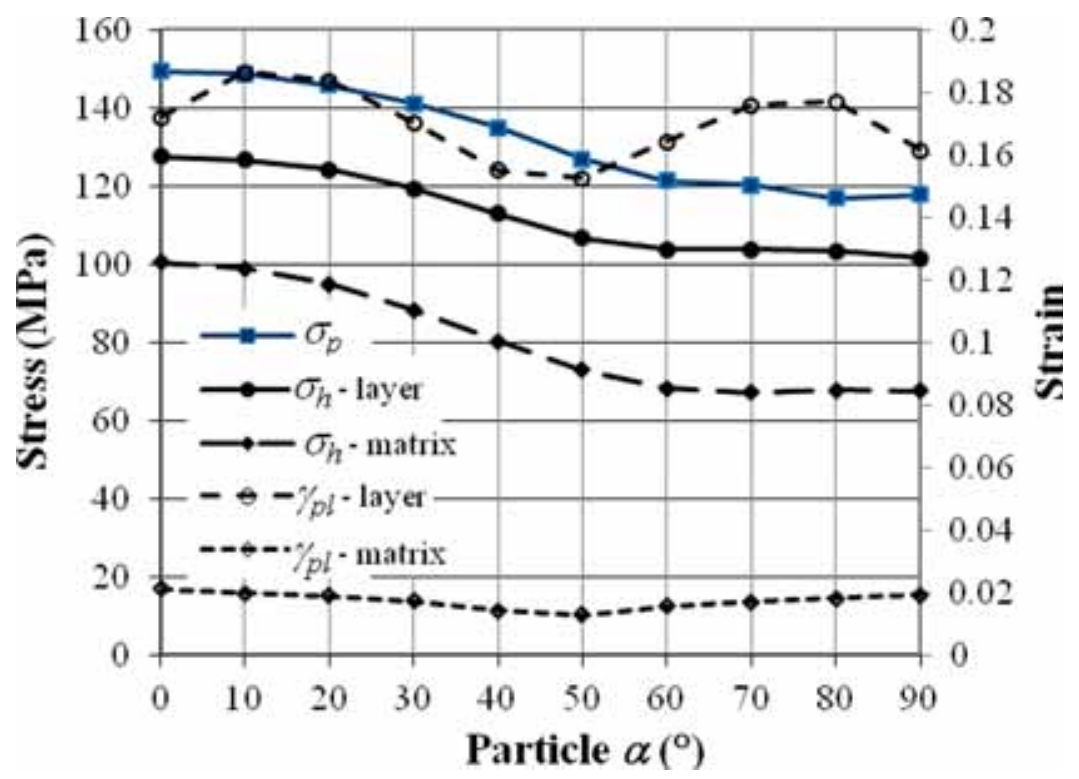

Figure 11: Critical stresses and strains versus particle orientation $(T=100 \mathrm{MPa}, r=2$, $t=0.02 b)$

the most critical. Only reliance on the present numerical predictions for $\gamma_{p l}$ would suggest that the two particle orientations mentioned above may favour fatigue crack initiation in the $S n$ layer due to cyclic plastic shear strain.

An interesting feature of the FBT measurements presented in Table 1 is the apparent strong link of fatigue initiation to the orientation $\left(\beta_{o}\right)$ of $S n$ layers. The mean of this angle drops from $0.93 \mathrm{rad}\left(53.3^{\circ}\right)$ for non-initiating layers to $0.50 \mathrm{rad}\left(28.6^{\circ}\right)$ for initiating ones. Hence, initiating layers are, on average, more closely aligned with the loading axis than the non-initiating ones. In the context of the present idealised micromechanical model, layer orientation coincides with that of the tangent to the elliptical particle boundary. Therefore, the consistency of experimental observations with analytical predictions was investigated by assessing the orientation of this tangent at the locations of maximum hydrostatic stress and plastic shear strain in the layer. As with the elastic analysis, the angular co-ordinates $\theta_{h}$ and $\theta_{\gamma}$ of these locations can be identified from the finite element output and the angles $\beta_{h}$ and $\beta_{\gamma}$ between the respective tangents and the loading axis determined from

$$
\beta=\tan ^{-1}\left(\frac{\tan \theta}{r}\right)+\alpha
$$

for a given aspect ratio $r$ and particle orientation $\alpha$. The predicted values of $\beta_{h}$ and $\beta_{\gamma}$ for the whole range of $\alpha$ as well as various $t /(2 b)$ ratios were examined and $\beta_{\gamma}$ was found consistently and significantly smaller than $\beta_{h}$, indicating that the layer orientation at the location of maximum plastic shear strain is closer to the loading direction. This can also be noted in the contour plots of Fig. 8(b) and (c); in this particular case, $\beta_{h} \cong 90^{\circ}$ while $\beta_{\gamma} \cong 40^{\circ}$. A comparison of such numerical predictions with experimental observations on the orientations of 
initiating and non-initiating Sn layers indicates a clear link between fatigue crack initiation and cyclic plastic shear strain.

\subsection{Effect of aspect ratio}

Figure 12 shows the effect of aspect ratio variation upon various important stress and strain components in the case of a particle embedded within an $S n$ layer of thickness equal to $2 \%$ of its major axis and $\alpha=0$. The maximum tensile stress within the particle rises with increasing $r$, but this appears not to be consistent with the FBT $r_{o}$ measurements for intermetallic particles, which show very little difference between initiating and non-initiating particles. However, it should be pointed out that $r_{o}$ is, by definition, always greater than 1 ; therefore, the entries of Table 1 (averages and standard deviations) have been calculated over a range of values that includes those of $r^{-1}$ for $r<1$. This means that any shift toward a higher $r_{o}$ average for the initiating particles is cancelled by the presence of particles with the safer $r^{-1}$ ratio, that is, particles with high $r_{o}$ but orientation closer to perpendicular to the loading direction.

Again, it is seen that the state of stress in the matrix remains less critical than that in the $S n$ layer. As $r$ increases, the hydrostatic stress in the layer also grows; this seems to suggest that void nucleation would be more likely for encapsulated particles with high aspect ratios and orientations close to the loading direction. In contrast, the maximum plastic shear strain in the layer appears to have an optimum at $r=1$. This indicates that, if fatigue initiation could be linked to cyclic plastic shear strain, then it would have been more likely with increasing $r_{o}$, irrespective of the particle orientation relative to the loading direction. There is, however, no direct link between hard particle aspect ratio and $S n$ layer failure in the present FBT results to confirm either of the two hypotheses.

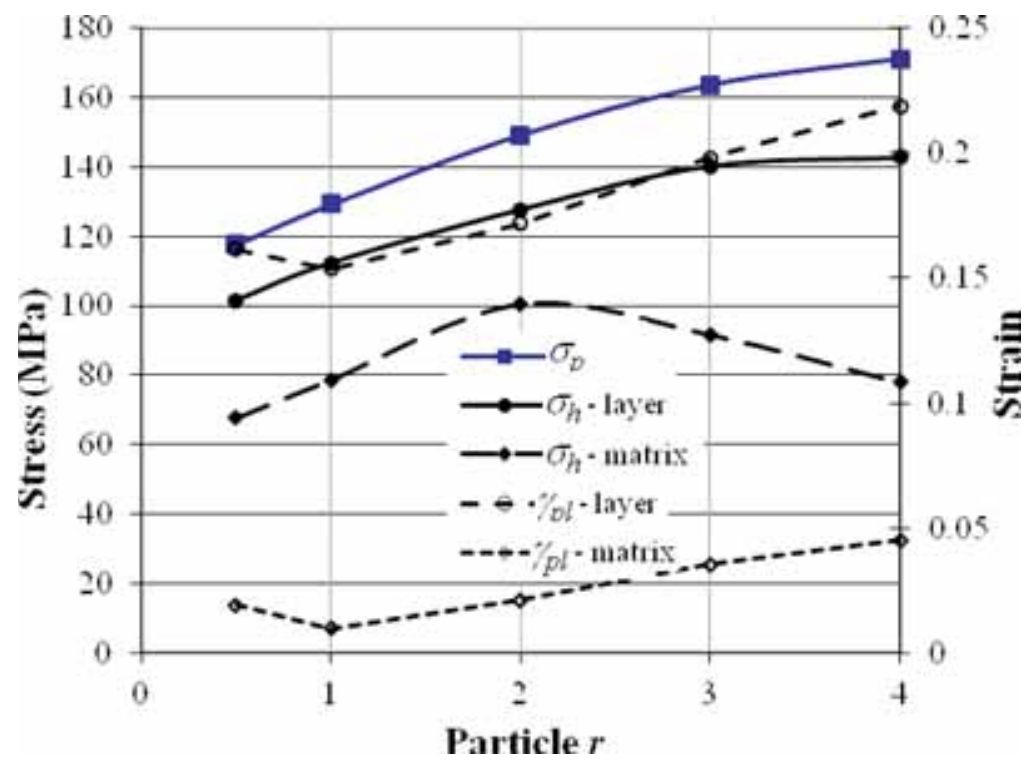

Figure 12: Variation of the critical stresses and strains with the $r$ of the particle $(T=100 \mathrm{MPa}$, $t=0.02 \times$ major axis, $\alpha=0^{\circ}$. 


\section{CONCLUSIONS}

The FBT measured features of the AS20S lining microstructure did not always follow well-defined trends that could be related to the size, shape, and orientation of the crack initiating particles compared to those previously obtained from the AS16 [2] and AS1241 [9] linings. Hence, the linking of these features to micromechanical analysis results was not a straightforward task. On the other hand, FE modelling was applied to ideal geometries; therefore, the obtained relations between various microstructural features for different phases and critical stress and strain components can only be linked qualitatively with the FBT measurements.

If fatigue initiation in the form of brittle fracture is explained in terms of high tensile stresses in the intermetallics, the results of the present micromechanical analysis lead to the expectation that fatigue failure would be more likely for (i) smaller $S n$ layer thickness relative to the particle major dimension, (ii) smaller object angles, and (iii) higher aspect ratios. It has been shown that the first two outcomes are consistent with the trends of the FBT measurements presented in Table 1; the third is not contradicted by the aspect ratio statistics for initiating and non-initiating particles, which are very similar due to the counterbalancing effect of non-initiating particles with high aspect ratios but orientations close to perpendicular to the loading direction. Particle size itself is a micromechanical feature clearly affecting fatigue initiation, and this observation applies to both soft $(S n)$ and hard (intermetallic, $S i)$ inclusions. For this effect to be investigated, a periodic or embedded cell micromechanical model is required. Such an investigation has already been carried out on the microstructure of the AS1241 bearing lining [9].

With regard to void nucleation or fatigue crack initiation within the $S n$ layers, two possible criteria can be used in the context of a micromechanical analysis: the maximum hydrostatic stress or the maximum plastic shear strain criterion. Consistency with experimental observations would indicate which one is the most relevant. With regard to Sn layer thickness, both criteria agree with experimental observations that very thin layers are more likely to detach. With regard to orientations more likely to cause fatigue initiation, the predictions of maximum plastic shear strain criterion appear to be more consistent with FBT measurements. Finally, the analysis predicted that rising object aspect ratios would increase the likelihood of failure according to both criteria, but the maximum hydrostatic stress criterion also requires object orientations close to the loading direction, while the maximum plastic shear strain criterion does not impose such a restriction. However, no relevant experimental observations were available for comparison and validation of either of these analytical outcomes.

Sizes, morphologies and distributions of the secondary-phase particles within the microstructure are dependent on a number of factors, which are related to their composition, manufacturing techniques, as well as a combination of various parameters used during mechanical working of the bearings and intermediate heat treatment. Fatigue crack initiation in such a material system was linked to microstructural features and critical stress and strain components through experimental observations and numerical modelling. The cracks, once initiated, were observed to grow consistently throughout a significant proportion of the fatigue life [3]. With the knowledge of optimum particle sizes and shapes, microstructural goals can be specified for one or more of these manufacturing and processing parameters to be adjusted to form an alloy with the best resistance to fatigue initiation.

\section{REFERENCES}

[1] Blount, E.A., Design factors influencing the fatigue resistance of connecting-rod big-end bearings. Proceedings of the Institution of Mechanical Engineers, 175(10), pp. 513-527, 1961. doi: http://dx.doi.org/10.1243/PIME PROC 196117503702 
[2] Mwanza, M.C., Joyce, M.R., Lee, K.K., Syngellakis, S. \& Reed, P.A.S., Microstructural characterisation of fatigue crack initiation in Al-based plain bearing alloys. International Journal of Fatigue, 25(9-11), pp. 1135-1145, 2003. doi: http://dx.doi. org/10.1016/S0142-1123(03)00148-8

[3] Ali, M.S., Reed, P.A.S. \& Syngellakis, S., Comparison of fatigue performance of HVOF spray coated and conventional roll bonded aluminium bearing alloys. Materials Science and Technology, 25(5), pp. 575-581, 2009. doi: http://dx.doi.org/ $\underline{10.1179 / 174328408 X 322213}$

[4] Kim, W.H. \& Laird, C., Crack nucleation and stage-I propagation in high strain fatigue. 2. mechanism. Acta Metallurgica, 26(5), pp. 789-799, 1978. doi: http://dx.doi. org/10.1016/0001-6160(78)90029-9

[5] Bowles, C.Q. \& Schijve, J., Role of inclusions in fatigue crack initiation in an Aluminum-alloy. International Journal of Fracture, 9(2), pp. 171-179, 1973. doi: http://dx.doi.org/10.1007/BF00041859

[6] Christian, K.D. \& German, R.M., Relation between pore structure and fatigue behavior in sintered Iron-Copper-Carbon. International Journal of Powder Metallurgy, 31(1), pp. 51-61, 1995.

[7] Kung, C.Y. \& Fine, M.E., Fatigue crack initiation and microcrack growth in 2024-T4 and 2124-T4 Aluminum-alloys. Metallurgical Transactions A-Physical Metallurgy and Materials Science, 10(5), pp. 603-610, 1979. doi: http://dx.doi.org/10.1007/ $\underline{B F 02658324}$

[8] Couper, M.J., Neeson, A.E. \& Griffiths, J.R., Casting defects and the fatigue behavior of an Aluminum casting alloy. Fatigue \& Fracture of Engineering Materials \& Structures, 13(3), pp. 213-227, 1990. doi: http://dx.doi.org/10.1111/j.1460-2695.1990.tb00594.x

[9] Joyce, M.R., Lee, K.K., Syngellakis, S. \& Reed, P.A.S., Quantitative assessment of preferential fatigue initiation sites in a multi-phase aluminium alloy. Fatigue \& Fracture of Engineering Materials \& Structures, 27(11), pp. 1025-1036, 2004. doi: http://dx.doi. org/10.1111/j.1460-2695.2004.00815.x

[10] Ali, M.S., Reed, P.A.S., Syngellakis, S., Moffat, A. \& Perrin, C., Microstructural factors affecting fatigue initiation in various Al based bearing alloys. Materials Science Forum, 519-521, pp. 1071-1076, 2006. doi: http://dx.doi.org/10.4028/www.scientific. net/MSF.519-521.1071

[11] Eshelby, J.D., The determination of the elastic field of an ellipsoidal inclusion and related problems. Proceedings of the Royal Society of London, A241, pp. 376-396, 1957. doi: http://dx.doi.org/10.1098/rspa.1957.0133

[12] Boselli, J., Pitcher, P.D., Gregson, P.J. \& Sinclair, I., Secondary phase distribution analysis via finite body tessellation. Journal of Microscopy-Oxford, 195, pp. 104-112, 1999. doi: http://dx.doi.org/10.1046/j.1365-2818.1999.00483.x

[13] Nutt, S.R. \& Duva, J.M., A failure mechanism in Al-SiC composites. Scripta Metallurgica, 20(7), pp. 1055-1058, 1986. doi: http://dx.doi.org/10.1016/00369748(86)90435-7

[14] Nutt, S.R. \& Needleman, A., Void nucleation at fiber ends in Al-SiC composites. Scripta Metallurgica, 21(5), pp. 705-710, 1987. doi: http://dx.doi.org/10.1016/00369748(87)90389-9

[15] Christman, T., Needleman, A. \& Suresh, S., An experimental and numerical study of deformation in metal ceramic composites. Acta Metallurgica, 37(11), pp. 3029-3050, 1989. doi: http://dx.doi.org/10.1016/0001-6160(89)90339-8 
[16] Whitehouse, A.F. \& Clyne, T.W., Critical stress criteria for interfacial cavitation in MMCs. Acta Metallurgica Et Materialia, 43(5), pp. 2107-2114, 1995. doi: http:// dx.doi.org/10.1016/0956-7151(94)00375-R

[17] Hardiman, N.J., Elliptic elastic inclusion in an infinite elastic plate. Quarterly Journal of Mechanics and Applied Mathematics, 7(2), pp. 226-230, 1954. doi: http://dx.doi. org/10.1093/qjmam/7.2.226

[18] MatWeb Material Property Data, available at http://www.matweb.com/search /PropertySearch.aspx

[19] Shi, D., Wen, B., Melnik, R., Yao, S. \& Lia, T., First-principles studies of Al-Ni intermetallic compounds. Journal of Solid State Chemistry, 182(10), pp. 2664-2669, 2009. doi: http://dx.doi.org/10.1016/j.jssc.2009.07.026

[20] Ali, M.S., Microstructural Modelling of Fatigue in Layered Bearing Architectures, PhD Thesis, University of Southampton, 2007.

[21] ANSYS, Inc., ANSYS Academic Research, Canonsburg, PA 15317, available at www. ansys.com

[22] Starink, M.J. \& Syngellakis, S., Shear lag models for discontinuous composites: fibre end stresses and weak interface layers. Materials Science and Engineering A-Structural Materials Properties Microstructure and Processing, 270(2), pp. 270-277, 1999. doi: http://dx.doi.org/10.1016/S0921-5093(99)00277-4 